\title{
Classical Unified Field Theory Relating to Electricity and Mass
}

\author{
Yougang Feng \\ College of Science, Guizhou University, Guiyang, China \\ Email:ygfeng45@aliyun.com
}

Received 15 March 2015; accepted 9 April 2015; published 10 April 2015

Copyright (C) 2015 by author and Scientific Research Publishing Inc.

This work is licensed under the Creative Commons Attribution International License (CC BY). http://creativecommons.org/licenses/by/4.0/

(c) $\underset{\mathrm{EY}}{\mathrm{B}}$ Open Access

\section{Abstract}

A classical unified field theory relating to electricity and mass is proposed. It indicates that uncertainty relations are the intrinsic attributes of space-time, and the law of the uncertainty quantities' change can be described by the potential functions of the space-time. The electromagnetic field is a stimulated state of the space-time, associated with electricity; its behaviors exhibit the properties of the positive potentials $\varphi$ and $\vec{A}$. The gravitowagnetic field is another stimulated state of the space-time related to mass; its properties are governed by the negative potentials $-\varphi$ and $-\vec{A}$. The gauge invariance and the symmetries of the set of $\varphi$ and $\vec{A}$ and the set of $-\varphi$ and $-\vec{A}$ determine mostly the interaction forms for the fields, and there are some corresponding physical properties between the two kinds of fields, including the weak interactions due to the spontaneous symmetry breaking of the potential functions. The discovery of the wagnetic field reminds us that there may be additional interactions beyond the conventional forms. Our theory provides an available foundation for the grand unified theory.

\section{Keywords}

\section{Uncertainty, Space-Time, Electricity, Mass, Potential Function, Weak Interaction}

\section{Introduction}

Physicists have been studying on physics in two categories: cosmology associated with general relativity and particle physics referring to quantum physics. Usually, they have to face on four types of forces: gravitation, electromagnetic forces, weak force, and strong force, no matter which subject they are concerned in. The unified theory of the weak and electromagnetic forces together with quantum chromodynamics to describe the strong force constitutes what physicists call the "Standard model" [1]-[3]. A grand unified theory is to combine all 
three of the forces. At present, theoretical workers are making effort to find a theory that includes gravity in a way that is consistent with quantum theory. An obvious difficulty is that Einstein's general relativity seems can't be quantization leading to that there is so for not a quantum theory for the gravitation.

In our opinion quantum behaviors should be hidden in the classical world. The road to the quantization of a classical field theory starts with the field equations, by which a lagrangian, via Hamilton's principle, will be found to represent them. Using the lagrangian it is then possible to identify canonical model and carry out the quantization to set up a quantum field. Clearly, there is no applicable classical field there is no effective quantum field. On one hand, according to special relativity the three components of momentum and the energy as the time component transform together to make what we call a "four-vector". The vector is tangent to the path in the position-time (x, y, z, t), which means that the momentum-energy and the space-time are dual to each other. On the other hand, their relevant components obey uncertainty relations [4]. We therefore can say that the uncertainty principle is available to those systems that are covariant in Lorentz transformation. Conversely, a classical system having covariance can be made quantization.

In terms of differential geometry differential manifolds are generalizations of Euclidean spaces. Any given point in a manifold has a neighborhood which is homeomorphic to an open set of a Euclidean space. Hence, we can establish local coordinates in a neighborhood of every point. A manifold is then consequence of pasting together pieces of a Euclidean space (a flat space). This is the reason why the general relativity is locally covariance [5]. The general relativity makes the gravitation be geometrilization, neglecting its physical origin and substantial property. Generally, the space-time represented by the theory is warped, in which the uncertainty principle fails to practice. Therefore, the theory is globally not covariant and can't be quantization. We think that the Einstein's matte painting for the gravitation is only an individual approach but a unique answer. We can set up a new classical theory for the fields subject to mass, and try to make the representation be covariant to Lorentz transformation like the Maxwell's theory. We have proved that the uncertainty relations are the intrinsic attributes of the space-time and the moment-energy rather than particle's properties [4]. We then realize that the wavefunctions do describe such attributes, because of which the wavefunctions can be determined from the classical dynamic equation, and consistent with the result coming from quantum theory. We also notice that the wavefunctions of light are just the positive potential functions of the free electromagnetic field, which reminds us that the wavefuctions of a free particle may be just the positive potential functions of the space-time in which the particle moves freely.

Quantum field theory portrays that the vacuum is the ground state of the relativistic quantum field, all possible fluctuation wavelengths are allowed for the electromagnetic fields. It is apparent that the fluctuations should obey uncertainty principle and the fluctuations' law can be described by the potential functions, which illustrate wave characteristics and covariance in Lorentz transformation as shown by electromagnetic field theory. It can be seen that the uncertainty relations and the potential functions always exist together no matter whether the space-time is stimulated or not. In this sense we may say that the uncertainty relations and the potential functions belong to the space-time and momentum-energy systems.

In Section 2 we propose that a stimulated space-time related to mass has two fields: gravitational field and wagnetic field, here the new word "wagnetic" is created by us to indicate the classical field determined by the rotation field of the negative vector potential. The properties of the gravitowagnetic fields involving the two fields are predicted in detail. In Section 3 Einstein's principle of equivalence, light track in the universe, superfluid Helium climbing walls effect, and antimatter are discussed by our theory. Section 4 is conclusion remark.

\section{Theory}

\subsection{Two Sets of Potential Functions}

The so called vacuum is the space-time in the ground state. Diract suggested early that a perfect vacuum was a region where all the states of positive energy were unoccupied and all those of negative energy were occupied [6]. He omitted the symmetry of the vacuum. In 1949 Feynman considered firstly the symmetric property of the space-time in that the negative energy states moved backwards in time correspond to positive energy antiparticles moving forwards in time [7]. With the development of quantum theory scientists have been increasingly attaching importance to the space-time symmetries, which conservation or breaking results directly in the creation or annihilation of a particle or a field and their relevant forces. In our opinion vacuum has the highest degree of symmetries, any point in which can be selected as an origin of a coordinate system, any direction at the point 
can be viewed as a position of the $\mathrm{x}$-axis, and there are uncertainty relations in such coordinate chart. The evolution law of the relevant uncertainty quantities depends on the potential functions (the scalar potential and the vector potential). The simplest symmetry of the potentials one can visualize is there are negative potential functions $-\varphi$ and $-\vec{A}$ together with the existence of the positive potential functions $\varphi$ and $\vec{A}$. It is expected under this circumstance that if there is a fluctuation distribution, there is also its opposite response. Vacuum is not absolutely quiet and the zero-point fluctuations caused by the uncertainty principle are subtle to keep the space-time to be in the ground state. On the contrary, a stimulated space-time has a definite coordinate system, while a lot of symmetries disappear.

For electricity and mass none of them has a priority to correspond to a stimulated space-time with occurrence of fields. Now that electromagnetic fields relating to the electricity is a type of the stimulated space-time, which can be represented by the functions $\varphi$ and $\vec{A}$; the stimulated space-time linking to mass should be demonstrated by the rest potential functions $-\varphi$ and $-\vec{A}$. The properties of the negative potentials $-\varphi$ and $-\vec{A}$ are exhibited by the behaviors of the gravitowagnetic fields including gravitation field and wagnetic field.

\subsection{Equations of Gravitowagnetic Fields}

The dynamic garavitation field intensity $\vec{R}$ is defined as

$$
\vec{R}=G\left[-\nabla(-\varphi)-\frac{1}{c} \frac{\partial(-\vec{A})}{\partial t}\right]=G\left(\nabla \varphi+\frac{1}{c} \frac{\partial \vec{A}}{\partial t}\right)
$$

Here the gravitation constant $G$ is introduced so as to unify the physical dimension, $\nabla \varphi$ is the gradient of $\varphi$. The wagnetic field intensity $\vec{W}$ is defined as

$$
\vec{W}=G \cdot \nabla \times(-\vec{A})=-G \cdot \nabla \times \vec{A}
$$

Here $\nabla \times \vec{A}$ is the rotation of $\vec{A}$. The wagnetic field is a field without sources, and the gravitational field is a field with sources. We introduce gravitowagnetic scalar potential and vector potential:

$$
\varphi_{g}=G \varphi, \quad \vec{A}_{g}=G \vec{A}
$$

Both potentials obey the following equations

$$
\begin{aligned}
& \nabla^{2} \varphi_{g}-\frac{1}{c^{2}} \frac{\partial^{2} \varphi_{g}}{\partial t^{2}}=4 \pi G \rho_{g} \\
& \nabla^{2} \vec{A}_{g}-\frac{1}{c^{2}} \frac{\partial^{2} \vec{A}_{g}}{\partial t^{2}}=4 \pi G \frac{\rho_{g} \vec{u}}{c}
\end{aligned}
$$

Here $\nabla^{2}$ is Laplace operator, $\rho_{g}$ is the mass density, $\vec{u}$ is the flow velocity of the mass, and $\rho_{g} \vec{u}$ gives the corresponding current density. All these functions in the equations are considered continuously varying functions of the space and time coordinates. We therefore have the equation of continuity, expressing the conservation of mass:

$$
\nabla \cdot\left(\rho_{g} \vec{u}\right)+\frac{\partial \rho_{g}}{\partial t}=0
$$

For the gravitostatic field, we get by Equation (4)

$$
\nabla^{2} \varphi_{g}=4 \pi G \rho_{g}
$$

This formula conforms with Newtonian attraction force law. The gravitowagnetic potentials, like the electromagnetic potentials consist with Lorentz gauge condition. Equations (4) and (5) indicate that the gravitowagnetic potentials propagate in light velocity although an object with mass, which is the field source, may move in a slow speed. For the free gravitowagnetic fields the right sides of Equations (4) and (5) vanish, the equations 
become standard wave equations like the free electromagnetic fields. The probability distribution of the relevant uncertainty quantities for the fields obeys the wave equations.

A free neutron corresponds to a gravitowagnetic field, the gravitation potential may act as wavefunction. A free electron bears relations to a free electromagnetic field and a free gravitowagnetic field, its wavefunction can be the linear superposition of the gravitation potential and the positive potential, or alternatively, either one of them (both synchronously change). A free electron is considered in reference [4], its electricity is omitted, thus the wavefunction is just the gravitation potential. It should be noticed that the wave velocity in the Equation (35) of the reference [4] is just light velocity. This example suggests that the potential function for a free field can be derived from the classical dynamic equation.

\subsection{Properties Arising from Gauge Invariance and Symmetries}

Since the gauge invariance determines mostly relevant forms of interactions and the set of the positive potential functions and the set of the negative potential functions are symmetric, the electromagnetic fields and the gravitowagnetic fields have similar properties in physics, there are some correspondence relations between the two kinds of fields:

The electrostatic field and the gravitostatic field are conservative.

The gradient of $\varphi$ leads to the principle of "charges repelling and opposite attracting" for the electronic force, the gradient of $-\varphi$ leads to the principle of "charges attracting" for the gravitation.

Both magnetic field and wagnetic field are rotation fields, and their lines of forces are closed.

Both electromagnetic fields and gravitowagnetic fields obey Lorentz transformation and they are covariant.

The essence of the spontaneous symmetry breaking in electromagnetic fields can be assigned as the positive potentials' one, such that the spontaneous symmetry breaking of the negative potentials' will lead to a weak interaction relative to mass. This inference is basically in agreement with the prediction about the weak interaction force being in company with massive mass proposed by quantum theory [8].

Chiral symmetry: if right-handed screw rule holds in the electromagnetic fields, the corresponding left-handed screw rule will appear in the gravitowagnetic fields, vice versa.

Like Lorentz force in the electromagnetic fields, there may be a similar force in the gravitowagnetic fields.

A spinning particle may have spin wagnetic moment like an electron has spin magnetic moment; the electron may have both the moments.

Both kinds of forces are long-range; the electromagnetic forces are far greater than the gravitowagnetic forces since the gravitation constant is far smaller than one.

Gravitons: the bosons that proceed from the quantization of the free electromagnetic wave are photons, and the bosons from the quantization of the free gravitowagnetic wave are gravitons. There exist photons and gravitons together in the quantization of both the free fields for a free electron with mass and electricity, and the photon's energy is far greater than the graviton's. There may be many similar properties between the photons and the gravitons due to the same generation mechanism. For this reason, it may be difficult to distinguish the gravitons from the photons.

\section{Discussion}

\subsection{Inequivalence between Inertial Mass and Gravitational Mass}

Any object with a mass feels both actions from the gravitational field and the wagnetic field provided by another moving object, its inertial mass, therefore, is not equivalent to its gravitational mass. Einstein's principle of equivalence in his general relativity is an approximate hypothesis, losing sight of the wagnetic field. It is reported there are certainly difference between the two masses [9] [10].

\subsection{The Track of Light in the Universe}

When a light passes by a huge star in the universe, there are two forces acting on the light: the star's gravitational force and the force similar to Lorentz force coming from the wagnetic field of the rotating star. If the two forces are not always in a definite plane the light track will be a complex curve, but a simple bending arc. This prediction should be verified by experiment. 


\subsection{New Interpretation of the Superfluid Helium Climbing Walls}

There is a strong wagnetic field created by the rotating superfluid Helium, which is induced by the foreign wagnetic field attributed to the rotating Earth. The field in the central region of the container, in which the Helium resides, and the Earth's wagnetic field attract to each other. Since the wagnetic line of force is closed, the liquid's field near the inwalls of the container repels to the Earth's field. Its intensity there is so strong that not only it resists dramatically against the Earth's wagnetic field, but also the reaction from the Earth makes the liquid get rid of the Earth's gravitation and drives further the liquid upwards along the inwalls. At the same time the intensity outside walls of the container is weak because of the walls' shield [11].

\subsection{Antimatter}

It seems that the gravitowagnetic fields' equations do not reject the matter with negative mass and the equations show that the mass and the negative mass obey the principle of "opposite repelling". This regularity may explain why there is not negative mass in our world. There are six composing types: positive charge with mass, positive charge with negative mass, negative charge with mass, negative charge with negative mass, mass without charge, negative mass without charge. Some particles of these types are found, for example proton, positron, electron, neutron; some need to be verified or denied.

\section{Conclusion Remark}

We believe that there is no quantum world; there is only an abstract quantum physical description of particle's motion. There is no available classical field theory there is no effective quantum field theory since the quantum behaviors are hidden in the classical world. The classical unified field theory involving the electromagnetic forces and the gravitowagnetic forces, attaching the relevant weak interaction forces, provides an applicable way to set up the grand unified theory.

\section{References}

[1] Glashow, S.L. (1980) Reviews of Modern Physics, 52, 539. http://dx.doi.org/10.1103/RevModPhys.52.539

[2] Salam, A. (1980) Reviews of Modern Physics, 52, 525. http://dx.doi.org/10.1103/RevModPhys.52.525

[3] Weinberg, S. (1980) Reviews of Modern Physics, 52, 515. http://dx.doi.org/10.1103/RevModPhys.52.515

[4] Feng, Y.G. (2014) Natural Science, 6, 1149-1158. http://dx.doi.org/10.4236/ns.2014.614103

[5] Utiyama, R. (1956) Physical Review, 101, 1597-1607. http://dx.doi.org/10.1103/PhysRev.101.1597

[6] Dirac, P.A.M. (1958) The Principle of Quantum Mechanics. 4th Edition (Revised), Oxford University Press, Oxford, 272.

[7] Feynman, R.P. (1949) Physical Review, 76, 769-789. http://dx.doi.org/10.1103/PhysRev.76.769

[8] Drukier, A.K., Freese, K. and Spergel, D.N. (1986) Physical Review D, 33, 3495-3508. http://dx.doi.org/10.1103/PhysRevD.33.3495

[9] Koester, L. (1976) Physical Review D, 14, 907-909. http://dx.doi.org/10.1103/PhysRevD.14.907

[10] Schmiedmayer, J. (1989) Nuclear Instruments and Methods in Physics Research Section A: Accelerators, Spectrometers, Detectors and Associated Equipment, 28, 59-62. http://dx.doi.org/10.1016/0168-9002(89)90248-9

[11] Minkel, J.R. (2009) Scientific American. 20 February. http://www.scientificamerican.com/article/superfluid-can-climb-walls/ 